Supplementary Information

\title{
Aerosol-to-hydrosol sampling and
}

\section{simultaneous enrichment of airborne}

\author{
bacteria for rapid biosensing
}

Hyeong Rae Kim, Sanggwon An, Jungho Hwang*

School of Mechanical Engineering, Yonsei University, Republic of Korea.

*Correspondence should be addressed to: hwangjh@yonsei.ac.kr;

Tel.: 82-2-2123-2821 


\section{Preparation of ConA-coated Magnetic Particles (CMPs)}

MagnaBind carboxyl-derivatized beads (Product No. 21353, Thermo Scientific, USA), phosphate-buffered saline (PBS) (Product No. 28372, Thermo Scientific, USA), 1-ethyl-3-(3-dimethylaminopropyl)carbodiimide hydrochloride (EDC) (Product No. 22980, Thermo Scientific, USA), conjugation buffer (Product No. 28390, Thermo Scientific, USA), MagnaBind magnets (Product No. 21357, Thermo Scientific, USA), and ConA (L7647, Sigma-Aldrich, USA) were purchased for preparing CMPs. One milliliter of MagnaBind beads were washed thrice with $1 \mathrm{~mL}$ of PBS. The beads were magnetically separated and aspirated after each wash. Magnetic separation was performed perpendicular to gravity. ConA was dissolved in the conjugation buffer at a concentration of $8 \mathrm{mg} / \mathrm{mL}$. One milliliter of ConA was added to the washed MagnaBind beads, and the mixture was gently agitated. Ten milligrams of EDC was dissolved in 1 $\mathrm{mL}$ of conjugation buffer immediately before use. One-tenth of a milliliter of the EDC solution was added to the beads-ConA mixture, and the resulting mixture was gently agitated. The mixture was incubated for $30 \mathrm{~min}$ at room temperature. To separate the ConA-coupled beads from the solution, a MagnaBind magnetic separation unit was used. The supernatant was aspirated from the coupled beads. Thereafter, the beads were washed thrice with $1 \mathrm{~mL}$ PBS. Finally, the CMPs for hydrosol-to-hydrosol enrichment of the collected bacteria were obtained. 


\section{Experimental evaluation of adsorption efficiency and recovery of test bacteria} during hydrosol-to-hydrosol enrichment by using the colony-counting method

Adsorption efficiency $\left(\eta_{\text {adsorption }}\right)$ is defined as follows:

$$
\eta_{\text {adsorption }}=1-\frac{C F U_{\text {After channel }}}{C F U_{\text {Before channel }}}
$$

where $C F U_{\text {Before channel }}$ is the colony number of bacteria that enter the enrichment channel, and $C F U_{A f t e r}$ channel is the colony number of bacteria that are not adsorbed onto the CMPs and exit the enrichment channel. For computing $\eta_{\text {adsorption, }}$ the prepared bacterial solution was spread on agar plates and incubated at $37{ }^{\circ} \mathrm{C}$ for $24 \mathrm{~h}$ $\left(C F U_{\text {Before channel }}\right)$. The same bacterial solution was passed through the enrichment channel, in which the CMPs were immobilized. The bacterial solution that was not adsorbed to the CMPs and subsequently exited the channel was spread onto the agar plates and incubated at $37^{\circ} \mathrm{C}$ for $24 \mathrm{~h}\left(C F U_{A f t e r ~ c h a n n e l}\right)$.

Recovery $(\mathrm{R})$ is defined as follows:

$$
R=\frac{C F U_{\text {recovery }}}{C F U_{\text {Before channel }}-C F U_{\text {After channel }}}
$$

where $C F U_{\text {recovery }}$ is the colony number of bacteria measured downstream of the enrichment channel when the magnetic blocks were removed after hydrosol-to-hydrosol enrichment. The concentrations of the bacterial samples used in the adsorption efficiency and recovery experiment were $10^{5}-10^{6} \mathrm{CFU} / \mathrm{mL}$ for all tested bacterial species. 
Table S1

Bacteria-CMPs adsorption efficiency.

\begin{tabular}{|c|c|c|c|c|}
\hline & S. aureus & E. coli & A. baumannii & B. cereus \\
\hline $\begin{array}{c}\text { Adsorption } \\
\text { efficiency }\end{array}$ & $0.30 \pm 0.02$ & $0.28 \pm 0.03$ & $0.29 \pm 0.03$ & $0.28 \pm 0.04$ \\
\hline
\end{tabular}

Table S2

Recovery rate of each enriched bacterium with different final volumes of liquid.

\begin{tabular}{|c|c|c|c|c|}
\hline Bacteria & $100 \mu \mathrm{L}$ & $20 \mu \mathrm{L}$ & $10 \mu \mathrm{L}$ & $5 \mu \mathrm{L}$ \\
\hline S. aureus & 0.99 & $0.78 \pm 0.05$ & $0.72 \pm 0.06$ & $0.69 \pm 0.09$ \\
\hline E. coli & 0.99 & $0.81 \pm 0.15$ & $0.75 \pm 0.11$ & $0.69 \pm 0.10$ \\
\hline A. baumannii & 0.99 & $0.76 \pm 0.21$ & $0.74 \pm 0.14$ & $0.66 \pm 0.15$ \\
\hline B. cereus & 0.99 & $0.81 \pm 0.14$ & $0.74 \pm 0.20$ & $0.67 \pm 0.14$ \\
\hline
\end{tabular}

Table S1 shows the adsorption efficiencies of each of the bacterial species onto the CMPs, as calculated using equation S1. The results show that the adsorption efficiencies of all bacteria are similar. This result is consistent with the results of a previous study, which showed that the maximum bacteria-to-ConA adsorption efficiencies of four species of bacteria (S. aureus, E. coli, B. cereus, and S. enteria) were similar (Kwon et al., 2019). ${ }^{1}$ Moreover, the small standard deviation values confirmed that the adsorption efficiencies of all bacteria were stable.

Table $\mathrm{S} 2$ shows the recovery rates $(\mathrm{R})$ of all the bacterial species with different final volumes of liquid used to flush the enrichment channel. The aerosol-to-hydrosolenriched but not hydrosol-to-hydrosol-enriched bacterial sample showed 0.99 recovery for all bacteria. This means that $99 \%$ of the aerosol-to-hydrosol-collected bacteria was recovered, and only $1 \%$ of the bacteria was lost in the channel. As the volume of 
flushing liquid decreased, the recovery rate decreased for all bacteria. However, even 5 $\mu \mathrm{L}$ of the flushing liquid recovered a large proportion of the enriched bacteria.

Although the trends of adsorption efficiency and recovery were confirmed, they may not be applicable to biosensors (electrical current measurement, fluorescence microscopy, and real-time qPCR) in this study because the commercial magnetic particles used in this study had an antibacterial characteristic owing to their iron (Fe)based composition. Iron causes bacterial cell death, especially in oxygen-rich environments. Therefore, iron can damage the cell membranes that protect intracellular materials and prevent the formation of bacterial colonies on agar plates (Dixon and Stockwell, 2014). ${ }^{2}$ Therefore, different results might be obtained with other biosensors. 


\section{Real-time qPCR amplification curves obtained in hydrosol-hydrosol enrichment}

\section{performance test.}
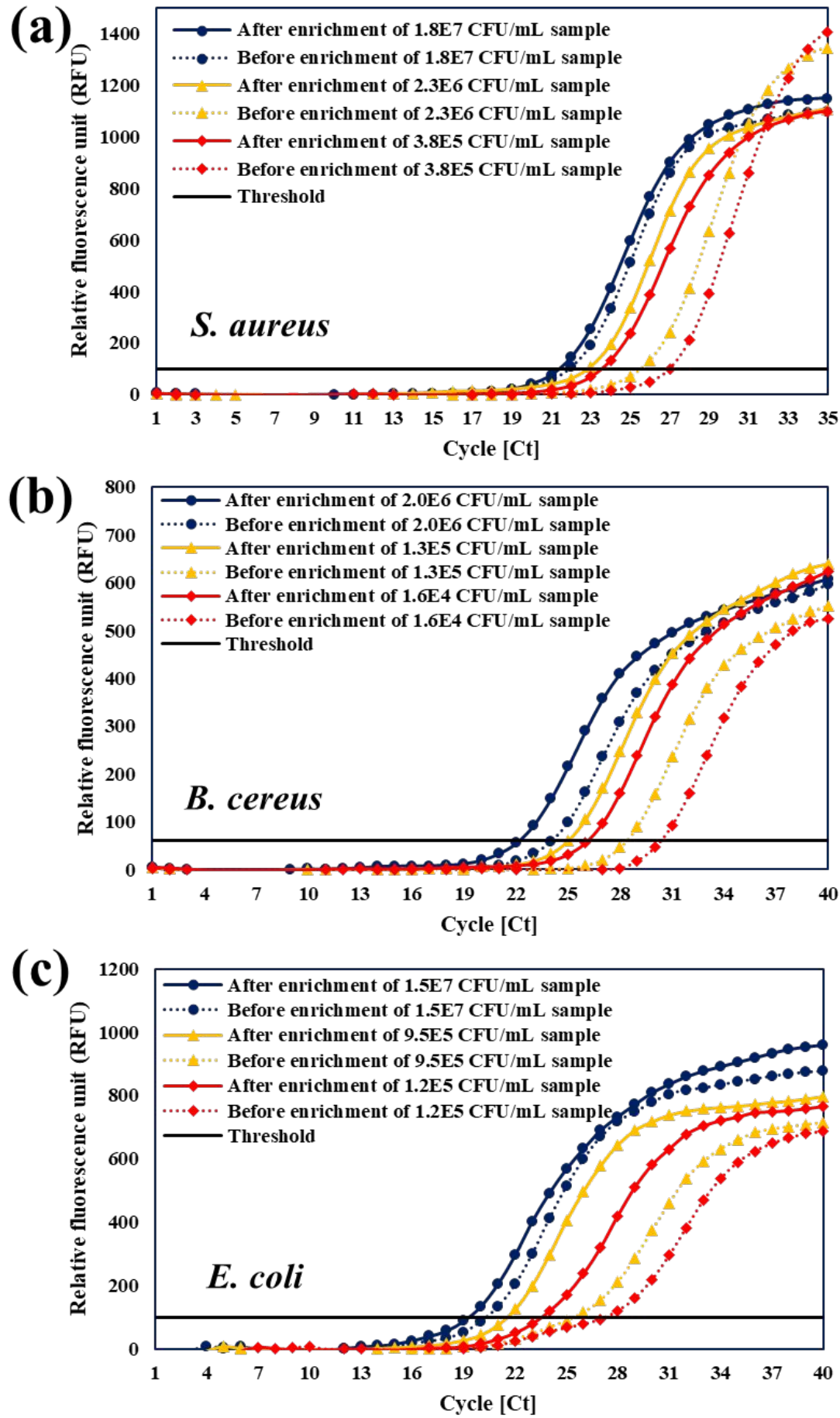

Figure S1. Real-time qPCR amplification curves: aerosol-to-hydrosol collected and after hydrosol-to-hydrosol enrichment for different concentrations of (a) $S$. aureus, (b) B. 
cereus, and (c) E. coli samples. The dotted curves that represent aerosol-to-hydrosol collected samples turned into continuous curves after hydrosol-to-hydrosol enrichment (i.e., the red dotted curves of aerosol-to-hydrosol collected sample turned into red continuous curves after hydrosol-to-hydrosol enrichment). 


\section{REFERENCES}

(1) Kwon, K.; Gwak, H.; Hyun, K. A.; Kwak, B. S.; Jung, H. I. Highthroughput microfluidic chip for magnetic enrichment and photothermal DNA extraction of foodborne bacteria. Sens. Actuator B: Chem. 2019, 294, 62-68.

(2) Dixon, S. J.; Stockwell, B. R. The role of iron and reactive oxygen species in cell death. Nat. Chem. Biol. 2014, 10(1), 9-17. 